Editorial for EBMH (February 2019 issue)

\title{
Providing the most appropriate care to our individual patients
}

\author{
Andrea Cipriani, Anneka Tomlinson
}

Department of Psychiatry, Warneford Hospital, University of Oxford, Oxford, UK; Oxford Health NHS Foundation Trust, Warneford Hospital, Oxford, UK

\author{
Corresponding author: \\ Professor Andrea Cipriani, \\ Department of Psychiatry, \\ Warneford Hospital, \\ University of Oxford, \\ Oxford OX3 7JX, \\ UK
}


With 350 million people affected in the world, depressive disorder is one of the top 20 causes of the overall global burden of disease. ${ }^{1}$ The high costs, both direct and indirect, of major depressive disorder are largely due to the significant deficits in treatment provision; ${ }^{2}$ and therefore remediable with current therapies. A key international challenge is to determine how best to implement currently available and effective treatments.

There are a number of efficacious pharmacological and non-pharmacological interventions for depressive disorder. ${ }^{3}$ Antidepressant drugs are recommended and frequently used as first-line therapy for adults with moderate to severe depressive disorder, and in the UK about $80 \%$ of people in primary care receive an antidepressant prescription in the first year of diagnosis. ${ }^{4}$ However, a significant proportion of these prescriptions are for less than 30 days, while an adequate trial of antidepressants is generally recommended to be 6-8 weeks before changing or stopping the medication. ${ }^{3} \mathrm{~A}$ too short duration of treatment limits both the therapeutic effect ${ }^{85}$ and increases the risk of withdrawal symptoms.

A number of factors contribute to the sub-optimal treatment duration of antidepressant drugs, the two most recognised contributing factors include the initial side effects of the medication and their perceived marginal efficacy. These factors are exacerbated by our current limited ability to predict which drug will causes the fewest undesirable side effects for a specific patient, and which will work most effectively. Improved methods to tailor specific treatments to individual patients are needed. Whilst the need for such an approach is widely recognised by NICE guidelines, this still remains an unmet clinical need, with no currently reliable ways of doing so. ${ }^{3}$

In fact, however, there are major opportunities to improve patient outcomes using existing therapies by the efficient use of available clinical trial data combined with the technical advances in data synthesis, and long-term outcomes from real-world datasets. ${ }^{56}$ Such analyses can now predict the probability of response for a specific subgroup of patients, or estimate the chances that a person will have a particular side effect. ${ }^{67}$ By matching individual patients to individual specific 
antidepressant drugs, clinicians can precisely customise treatment to patients' individual needs and preferences to ultimately improve their clinical outcome.

Internationally, precision medicine is now a leading aim of healthcare. ${ }^{78}$ Currently, the process of matching patients to treatments is too often by trial and error, delaying clinical improvement and increasing the risks and costs associated with ineffective treatment. Pooling an analysis of data from clinical trials may provide "personalised" estimates of comparative effectiveness, stratified for specific subgroups of patients to predict "individualised" response to treatment. ${ }^{8}$ Despite important progress in trying to identify depressed patients who may respond differently, ${ }^{9}$ psychiatry continues to lag behind other specialties like cardiology, oncology and stroke (where simple clinical variables were used to target aspirin and heparin to individual patients).

Factors influencing an individual's drug response in depressive disorder include environmental (e.g. co-medications, smoking and food), clinical (e.g. severity of illness, previous treatments) and personal or demographic variables (e.g. age, gender and family history). However, the wealth and variety of these factors creates its own challenges..$^{10}$ New methodologies and tools are needed which: ${ }^{15}$

- $\quad$ are based on robust evidence;

- $\quad$ are acceptable to patients and clinicians;

- guide and direct treatment personalisation, incorporating patients' views and values, and clinical judgement;

- support probabilistic decision-making. For instance, a difference in efficacy between interventions of, say, $5 \%$ might mean more to one patient than to another and it does not precisely exclude a benefit that clinicians and patients might find meaningful. Or, vice versa, the same result could allow some doctors and patients to choose to avoid the treatment after carefully considered tolerability, risk and uncertainty. 
When several treatment options are available, standard meta-analyses provide only partial information because they can only answer questions about pairs of treatments. ${ }^{11 \theta}$ This fragmented approach does not support optimal clinical decision making, ${ }^{121}$ and the need for a robust method to summarise evidence across several, indeed many, interventions has been increasingly recognised. ${ }^{1 \underline{3} z}$ Network meta-analysis has been developed to (1) synthesise evidence across a network of randomised trials, (2) allow the estimation of the relative effectiveness of several interventions and (3) produce ranked treatment options. ${ }^{1{ }^{143}} \mathrm{~A}$ further advancement is individual patient data network meta-analysis (IPD-NMA). ${ }^{154}$ IPD-NMA allows the comparative efficacy of different treatments to be assessed, as well as providing a prediction of the clinical outcome, looking for instance at time to response or temporal trajectory of side effects. This is a novel and exciting development which will hopefully drive forward evidence-based practice.

Treatment algorithms have contributed to advances in many fields of medicine including psychiatry, but studies have consistently shown that the initial benefits of algorithm implementation are not sustained once the implementation support is withdrawn. Computerised decision systems have been developed to provide ongoing assistance to clinicians. However, all algorithms developed to date lack the ability to apply the best knowledge directly to the individual patient and selectively provide information relevant to the characteristics and circumstances of that patient in their specific situation. This requires human interpretation and clinician-patient interaction. ${ }^{165}$ So, as we recently said, ${ }^{17 z}$ "is it now time to move away from a 'one size fits all approach' and move towards precision mental health, providing the most appropriate care to our patients individually?" 


\section{References}

1. GBD 2016 Disease and Injury Incidence and Prevalence Collaborators. Global, regional, and national incidence, prevalence, and years lived with disability for 328 diseases and injuries for 195 countries, 1990-2016: a systematic analysis for the Global Burden of Disease Study 2016. Lancet 2017; 390: 1211-59.

2. Docherty M, Shaw K, Goulding L, et al. Evidence-based guideline implementation in low and middle income countries: lessons for mental health care. Int J Ment Health Syst 2017;11:8.

3. National Institute for Health and Care Excellence (NICE). Depression in adults: recognition and management (NICE Guideline CG90). Available at: https://www.nice.org.uk/guidance/cg90.

4. Moore $\mathrm{M}$, Yuen HM, Dunn N, et al. Explaining the rise in antidepressant prescribing: a descriptive study using the general practice research database. BMJ 2009;339: b3999.

5. Batelaan NM, Bosman RC, Muntingh A, Scholten WD, Huijbregts KM, van Balkom AJLM. Risk of relapse after antidepressant discontinuation in anxiety disorders, obsessive-compulsive disorder, and post-traumatic stress disorder: systematic review and meta-analysis of relapse prevention trials. BMJ 2017;358:j3927.

65. Califf RM, Robb MA, Bindman AB, et al. Transforming Evidence Generation to Support Health and Health Care Decisions. N Engl J Med. 2016; 375: 2395-400.

76. Chekroud AM, Zotti RJ, Shehzad Z, et al. Cross-trial prediction of treatment outcome in depression: a machine learning approach. Lancet Psychiatry 2016; 3: 243-50.

87. Manrai AK, Patel CJ, loannidis JPA. In the Era of Precision Medicine and Big Data, Who Is Normal? JAMA 2018; 319: 1981-2.

89. Chekroud AM, Gueorguieva R, Krumholz HM, et al. Reevaluating the Efficacy and Predictability of Antidepressant Treatments: A Symptom Clustering Approach. JAMA Psychiatry 2017; 74: $370-8$. 
109. Koutsouleris N, Kahn RS, Chekroud AM, et al. Multisite prediction of 4-week and 52-week treatment outcomes in patients with first-episode psychosis: a machine learning approach. Lancet Psychiatry. 2016; 3: 935-46.

110. Efthimiou O. Practical guide to the meta-analysis of rare events. Evid Based Ment Health 2018; 21: 72-6.

121. Fusar-Poli P, Radua J. Ten simple rules for conducting umbrella reviews. Evid Based Ment Health 2018; 21: 95-100.

13zz. Mavridis D, Giannatsi M, Cipriani A, et al. A primer on network meta-analysis with emphasis on mental health. Evid Based Ment Health 2015; 18: 40-6.

1433. Leucht S, Chaimani A, Cipriani A, et al. Network meta-analyses should be the highest level of evidence in treatment guidelines. Eur Arch Psychiatry Clin Neurosci. 2016; 266: 477-80.

14․․ Furukawa TA, Efthimiou O, Weitz ES, et al. Cognitive-Behavioral Analysis System of Psychotherapy, Drug, or Their Combination for Persistent Depressive Disorder: Personalizing the Treatment Choice Using Individual Participant Data Network Metaregression. Psychother Psychosom 2018; 87: 140-153.

165. Henshall C, Marzano L, Smith K, et al. A web-based clinical decision tool to support treatment decision-making in psychiatry: a pilot focus group study with clinicians, patients and carers. BMC Psychiatry. 2017; 17: 265. 